\title{
Factors Influencing the Use of a Mobile App for Reporting Adverse Drug Reactions and Receiving Safety Information: A Qualitative Study
}

\author{
Sieta T. de $\operatorname{Vries}^{1}$ (D) Lisa Wong ${ }^{2}$ - Alastair Sutcliffe ${ }^{2} \cdot$ \\ François Houÿez ${ }^{3}$ - Carmen Lasheras Ruiz ${ }^{3}$ - Peter G. M. Mol ${ }^{1}$. \\ IMI Web-RADR Work Package 3b Consortium
}

Published online: 29 December 2016

(c) The Author(s) 2016. This article is published with open access at Springerlink.com

\begin{abstract}
Introduction A mobile app may increase the reporting of adverse drug reactions (ADRs) and improve the communication of new drug safety information. Factors that influence the use of an app for such two-way risk communication need to be considered at the development stage. Objective Our aim was to reveal the factors that may influence healthcare professionals (HCPs) and patients to use an app for two-way risk communication.

Methods Focus group discussions and face-to-face interviews were conducted in the Netherlands, Spain and the UK. Patients with type 2 diabetes mellitus, patients with a rare disease or their caregivers and adolescents with health conditions were eligible to participate. HCPs included pharmacists, paediatricians, general practitioners, internists, practice nurses and professionals caring for patients with a rare disease. Patients and HCPs were recruited through various channels. The recorded discussions and interviews were transcribed verbatim. The dataset was
\end{abstract}

The Web-RADR work package $3 \mathrm{~b}$ representatives are listed in Acknowledgements.

Electronic supplementary material The online version of this article (doi:10.1007/s40264-016-0494-x) contains supplementary material, which is available to authorized users.

Peter G. M. Mol

p.g.m.mol@umcg.nl

1 Department of Clinical Pharmacy and Pharmacology, University of Groningen, University Medical Center Groningen, Groningen, The Netherlands

2 Population, Policy and Practice Programme, UCL Institute of Child Health, 30 Guilford Street, London WC1N 1EH, UK

3 European Organisation for Rare Diseases (EURORDIS), Paris, France analysed using thematic analysis and arranged according to the Unified Theory of Acceptance and Use of Technology. Results Seven focus group discussions and 13 interviews were conducted. In total, $21 \mathrm{HCPs}$ and 50 patients participated. Identified factors that may influence the use of the app were the type of feedback given on reported ADRs, how ADR reports are stored and the type of drug news. Also mentioned were other functions of the app, ease of use, type of language, the source of safety information provided through the app, security of the app, layout, the operating systems on which the app can be used and the costs. Conclusions Further research is needed to assess associations between user characteristics and the direction (positive or negative) of the factors potentially influencing app use.

\section{Key Points}

Factors influencing the use of an app for two-way risk communication concern what feedback would be given on adverse drug reaction reports and how safely these are stored and handled; what type of news, if any, would be provided through the app; other functions of the app; ease of use; the source (authority) behind the app; appropriate language and appeal of the app; and costs.

User characteristics (e.g. age, education, experience with apps) may moderate to what extent specific factors (e.g. functions, security) influence use of the app.

When designing or improving an app for two-way risk communication, pharmacovigilance experts, and app researchers and developers should consider the identified factors. 


\section{Introduction}

Although the efficacy and safety of a drug has been assessed during the pre-marketing phase, post-marketing surveillance is particularly needed to complement the drug's safety profile [1-3]. In post-marketing surveillance, spontaneous reporting of adverse drug reactions (ADRs) to the national regulatory agency has been the leading method for decades [4]. Previously, ADRs were primarily reported by healthcare professionals (HCPs). Over time, interest in ADRs submitted directly by patients increased with the recognition of the added value of patient reports [5-7]. In many countries, patients are now allowed to report ADRs to spontaneous reporting systems [8]. A major limitation of spontaneous reporting, however, is that many ADRs go unreported. There are multiple reasons for this, especially time constraints and uncertainty about the causal relationship between an experienced symptom and a drug [9-14]. In addition, many patients state that they are not aware of the possibility of reporting an ADR to the national regulatory agency [10]. Moreover, patients may experience recall problems when too much time has passed between the onset of an ADR and reporting it [15].

Nevertheless, from time to time, spontaneously reported ADRs and other current post-marketing surveillance methods result in the detection of new safety signals that change the benefit-harm profile of the drug [16]. Subsequently, HCPs need to be informed. Throughout the EU, important new drug safety issues are primarily communicated by sending paper-based warning letters directly to HCPs [16]. In addition, many regulatory authorities publish these safety issues on their website. However, it is apparent from previous studies that new safety issues do not always reach the HCP nor affect clinical behaviour $[17,18]$. It is also recognised that patients need to be informed about the safety issues of drugs that are prescribed to them [19]. This promotes patient involvement, and informed and shared decision making in their treatment [20].

Recent technological developments such as mobile apps may increase ADR reporting and improve the communication of new safety issues. The combination of reporting ADRs and receiving safety information is known as twoway risk communication. An app developed for two-way risk communication may reduce the time between experiencing and reporting an ADR and may lead to more informed patients and HCPs. The Web-Recognizing Adverse Drug Reactions (Web-RADR) project (https:// web-radr.eu/) started with the development, testing and implementation of an app for two-way risk communication. The Web-RADR project aims to improve traditional pharmacovigilance activities by using new tools to identify potential new ADRs earlier (social media mining and the mobile app) and to improve drug safety communication (mobile app). The project has been described elsewhere in more detail [21]. The app developed for the Web-RADR project is intended for both patients and HCPs. In an app's development, involvement of end users is important to ensure that their opinions and values are taken into account. This will improve acceptance of the app [22]. Previous studies have been conducted to identify factors that may influence the use of mobile apps on, for instance, managing obesity [23] and diabetes mellitus [24] or preventing sexually transmitted infections and drug abuse in adolescents [25]. These studies show that app use will be influenced by factors such as ease of use, layout, and security of the data held in the app.

The aim of the current study was to identify factors which HCPs and patients suggested could influence their use of a mobile app for two-way drug risk communication. Findings from this study will be of interest to pharmacovigilance experts (regulatory agencies, industry and academics), and app researchers and developers.

\section{Methods}

\subsection{Study Design}

We performed a qualitative, multi-country study to identify the factors that may influence the use of an app for twoway risk communication in different patient and HCP groups (Table 1). The qualitative methods used were focus group discussions and face-to-face interviews. Countries involved were the Netherlands, Spain, and the UK. Ethical approval for this study was sought as determined by each country's local ethics committee policy. Informed consent was obtained from all participants.

\subsection{Population}

Patients with type 2 diabetes, patients with a rare disease (e.g. familial Mediterranean fever, narcolepsy) or their caregivers, and adolescents with health conditions were included in this study. These patient groups were selected to cover a wide variety of potential factors influencing the use of an app in a population that differed with respect to age, treatment options, and disease and treatment burden. An equally diverse group of HCPs serving these populations was recruited: pharmacists, professionals linked to patients with a rare disease, paediatricians, general practitioners (GPs), internists and practice nurses. Inclusion criteria for all participants were being able to speak, read and write the local language, owning a smartphone or tablet, being able to use such technology, and providing informed consent. In the case of adolescents aged $\leq 16$ 
Table 1 Methods per patient population

\begin{tabular}{|c|c|c|c|c|c|c|}
\hline Patients & HCPs & Country & Study design & Compensation & $\begin{array}{l}\text { Ethical } \\
\text { approval }\end{array}$ & Recording \\
\hline $\begin{array}{l}\text { Patients with } \\
\text { T2DM }\end{array}$ & $\begin{array}{l}\text { General } \\
\text { practitioners; } \\
\text { Internists; } \\
\text { Pharmacists; } \\
\text { Practice nurse }\end{array}$ & The Netherlands & $\begin{array}{c}\text { Face-to-face } \\
\text { interviews }\end{array}$ & $\begin{array}{l}\text { HCPs received a voucher of } € 75 \text { for } \\
\text { their participation. Patients received } \\
\text { a voucher of } € 50\end{array}$ & $\begin{array}{l}\text { UMCG } \\
\text { (reference } \\
\text { number } \\
\text { M15.172178) }\end{array}$ & Video \\
\hline $\begin{array}{l}\text { Patients with a } \\
\text { rare disease } \\
\text { and } \\
\text { caregivers of } \\
\text { such patients }\end{array}$ & $\begin{array}{l}\text { HCPs from the } \\
\text { Hospital Sant } \\
\text { Joan de Déu } \\
\text { from Barcelona }\end{array}$ & Spain & $\begin{array}{l}\text { Focus group } \\
\text { discussions }\end{array}$ & $\begin{array}{l}\text { Participants were compensated for } \\
\text { their time and travel, according to } \\
\text { the established budget for the } \\
\text { EURORDIS involvement in the IMI } \\
\text { Web-RADR project }\end{array}$ & $\begin{array}{c}\text { Fundació Sant } \\
\text { Joan de Déu }\end{array}$ & Audio \\
\hline Adolescents & $\begin{array}{l}\text { Lead Pharmacist } \\
\text { Medication } \\
\text { Safety; } \\
\text { Paediatricians; } \\
\text { Hospital doctors }\end{array}$ & UK & $\begin{array}{l}\text { Face-to-face } \\
\text { interview } \\
\text { and focus } \\
\text { group } \\
\text { discussions }\end{array}$ & $\begin{array}{l}\text { Adolescents were compensated travel } \\
\text { expenses and refreshments/lunch for } \\
\text { participating in the focus groups. A } \\
\text { thank-you letter was sent to each } \\
\text { adolescent following the session. } \\
\text { Paediatricians, hospital doctors and } \\
\text { pharmacists were not offered any } \\
\text { compensation }\end{array}$ & $\begin{array}{l}\text { UCL Research } \\
\text { Ethics } \\
\text { Committee }\end{array}$ & Audio \\
\hline
\end{tabular}

EURORDIS European Organisation for Rare Diseases, HCPs healthcare professionals, IMI Innovative Medicines Initiative, T2DM type 2 diabetes mellitus, UCL University College London, $U K$ United Kingdom, UMCG University Medical Center Groningen, Web-RADR WebRecognizing Adverse Drug Reactions

years, a parent or guardian had to provide informed consent. A specific inclusion criterion for patients was that they had ever experienced (or detected, in the case of caregivers) an ADR from any drug they (or their child) had used. Participants were recruited through various channels such as advertisements on websites and direct contact via patient and HCP organizations (Online Resource 1, see electronic supplementary material). Most of the participants received some form of compensation (Table 1).

\subsection{Procedure}

The focus group discussions and face-to-face interviews were led by one of the researchers (STdV in the Netherlands, CLR in Spain, and LW in the UK). Some of these researchers had previous experience with qualitative research and all attended a 1-day face-to-face training led by an expert in qualitative research. The discussions and interviews consisted of three steps (adapted from [26]): (1) an introduction, (2) discussion about factors potentially influencing the use of an app for two-way risk communication in general and (3) opinions about and influencing factors for a prototype version of the Web-RADR app. During the first step, participants completed a consent form and provided baseline characteristics: age, gender and educational level (for patients), or profession (specialisation of HCP). A semi-structured guide was used during the second and third step (Online Resource 2, see electronic supplementary material). On average, the duration of the focus group discussions and face-to-face interviews was approximately $90 \mathrm{~min}$. Focus group discussions and faceto-face interviews were conducted until theoretical saturation was reached. Theoretical saturation means that no new topics appear and all aspects of a theory are covered [27]. All data collected from the discussions and interviews across all countries were used in this assessment.

\subsection{Web-RADR App Prototype}

The prototype version of a mobile app for two-way risk communication was developed by the Web-RADR consortium for iOS and Android devices. The prototype was developed in English and translated to Dutch and Spanish versions for use in this study. To access the app, users had to enter an e-mail address and password on a login screen (Fig. 1a). The app contained two major functions: the ability to report ADRs (Fig. 1b) and to receive news on drug safety issues (Fig. 1c). In addition, users were able to view a graphical presentation of the number of reported ADRs per drug (Fig. 1d).

During the focus group discussions and face-to-face interviews, participants used the prototype app on a device provided by the researchers. In some cases (e.g. no internet connection), screenshots of the app were presented. In situations where the screenshots were used, this did not deter lively discussion. Participants were asked to go through the app and report any problems or issues they detected. These app-specific comments and suggestions have been reported 
Fig. 1 Screenshots of the UK version of the prototype app for two-way risk communication. a Login screen, $\mathbf{b}$ first screen to report an adverse drug reaction (ADR), $\mathbf{c}$ example of news items, $\mathbf{d}$ overview of reported ADRs

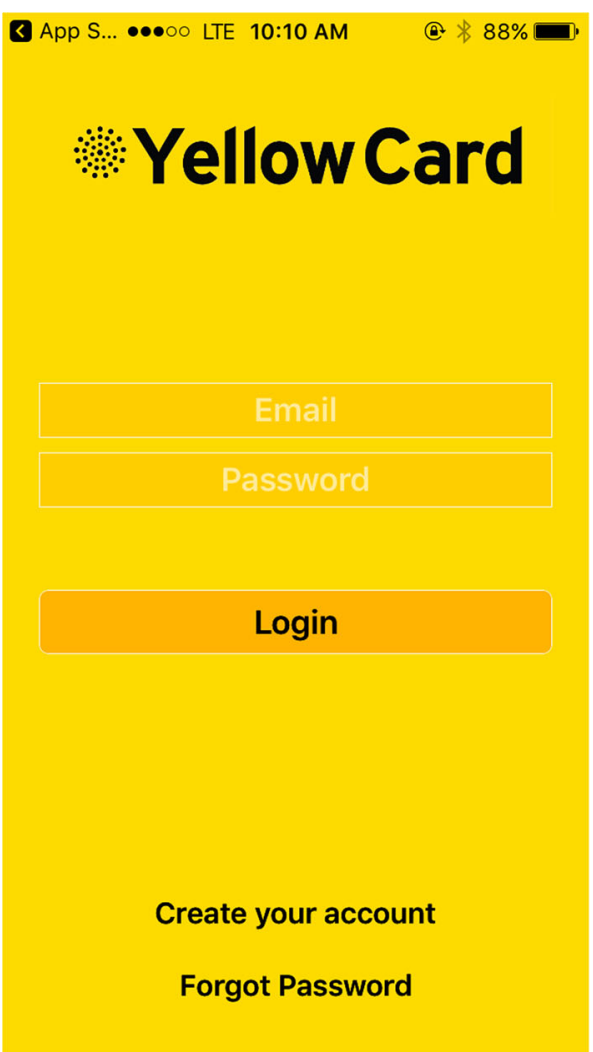

(A)

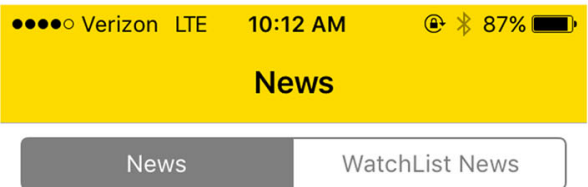

Field Safety Notices - 3107 Nov 2016 > October - 4 November

Summary List of field safety notices

(FSNs) from medical device

manufacturers from 31 October - 4 N...

Field Safety Notices - 2401 Nov 2016 > - 28 October 2016

Summary List of field safety notices (FSNs) from medical device

manufacturers from 24 - 28 October...

Medical device alerts 27 Oct 2016 > archived in October 2016

A list of all medical device alerts that were archived in October 2016.

\section{Total intravenous \\ 27 Oct $2016>$} anaesthesia sets (TIVA) -

Manufactured by Global Components

Medical Ltd - if propofol leaks from the check valve Luer lock connector pati..

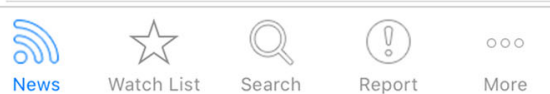

(C)

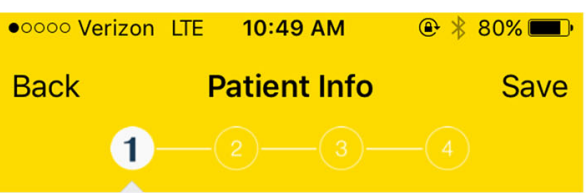

You are reporting this information as a Consumer or other non health

professional. If this is not correct, please

change your profile settings

PATIENT INFORMATION

Who experienced Please Select

\section{Gender: Gender}

Age:

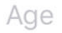

Initials:

Initials

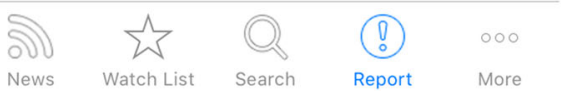

(B)

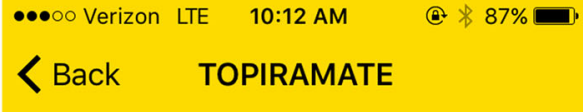

\section{Q) TOPIRAMATE}

\section{Nervous system \\ Psychiatric}

General \& administration site conditions

Investigations

Gastrointestinal

Skin \& subcutaneous tissue

Eye

499
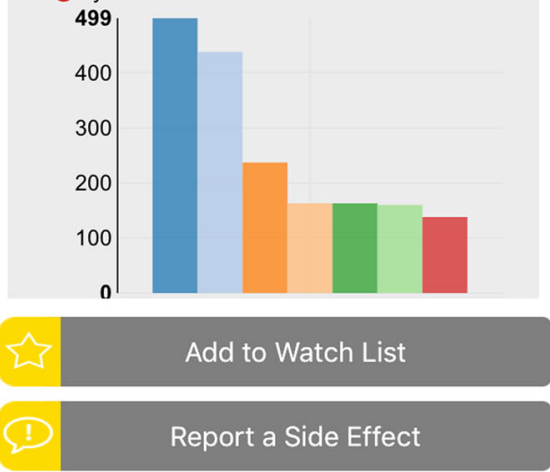

News

(D) 
to the Web-RADR consortium to improve the app. Following review of the app, the semi-structured guide was used to determine any additional factors that may influence the use of a mobile app for two-way risk communication based on the participants' experience with the prototype version of the app.

\subsection{Analyses}

The focus group discussions and face-to-face interviews were video- or audio-recorded (Table 1) and transcribed verbatim by one of the researchers. The transcripts were analysed by one researcher (Spain) or two researchers (the Netherlands and the UK) using thematic analysis in which themes or patterns were identified using a deductive (topdown)/theoretical approach [28]. The theoretical model used for this analysis was the Unified Theory of Acceptance and Use of Technology (UTAUT) [29]. This model states that factors related to performance expectancy, effort expectancy, social influence and facilitating conditions influence users' intention to use an app and whether they will actually use it [29]. In addition, gender, age, experience and voluntariness of use are described as moderating variables in the model. The analyses of the three countries were integrated into one overall report developed by one of the researchers and checked by all others to reduce any biases.

\section{Results}

In total, seven focus group discussions and 13 face-to-face interviews were conducted in which $21 \mathrm{HCPs}$ and 50 patients participated (Online Resource 3, see electronic supplementary material). Of the participating HCPs, ages ranged from 29 years old in the UK to 60 years old in Spain. Most of the HCPs were female (range from $50 \%$ in the Netherlands to $75 \%$ in the UK). The age of participating patients ranged from 11 years old in the UK to 67 years old in Spain. Most of the patients were female, ranging from $57 \%$ in Spain to $77 \%$ in the UK.

\subsection{Factors Influencing the Use of an App for Two- Way Risk Communication}

Several factors and moderating variables that may influence the use of an app for two-way risk communication were raised during the focus group discussions and face-toface interviews. A summary of these identified influencing factors and moderating variables is presented in Fig. 2. In the following sections, the identified influencing factors are described per theme of the UTAUT model.

\subsubsection{Performance Expectancy}

3.1.1.1 Feedback Participants indicated that it should be possible to provide feedback on the app.

“... make sure that there is something of, if necessary even a link in case the app doesn't work well; report this through this link. Or comments about the app; report this through this link. That people can mention it somewhere" (the Netherlands, Patient 4).

Most of the participants preferred some kind of feedback after submitting an ADR report. This ranged from a simple confirmation that the report was successfully sent, to an overview of how often this type of ADR has been reported earlier, to receiving feedback on what ultimately was done with the report (e.g. whether the report resulted in a new safety signal).

"Basically, what I would like to have is feedback what happened with the reports I have previously submitted through the app" (Spain, focus group with patients and their carers).

In a UK focus group with adolescents, concerns were raised that feedback might come too late if the ADR was serious. In this group a suggestion was made that a serious ADR could be flagged in the GPs' electronic prescribing system alerting them to contact the patient. [Of note, this is a function currently not foreseen for the app.]

Some participants, however, saw feedback as annoying. Suggestions were made to provide checkboxes where app users could indicate if they wish to receive feedback.

3.1.1.2 Storage of Reports Most of the participants preferred an option to access their earlier reported ADRs and to store unfinished reports to complete later.

3.1.1.3 News in the App There were some opposing views on news items in the app. Some participants felt that news could be useful.

"I think that this can be a fast way to inform endusers, prescribers about new information that is known about it" (the Netherlands, HCP 8).

However, others felt that there was already too much information

"There is so much information being constantly generated, that we are not able to take up such amount of information" (Spain, focus group with HCPs)

and that the app's goal should simply be to report ADRs

"I would not directly link it [drug safety information] to the app to my feeling, you have other sources for that" (the Netherlands, HCP 2). 


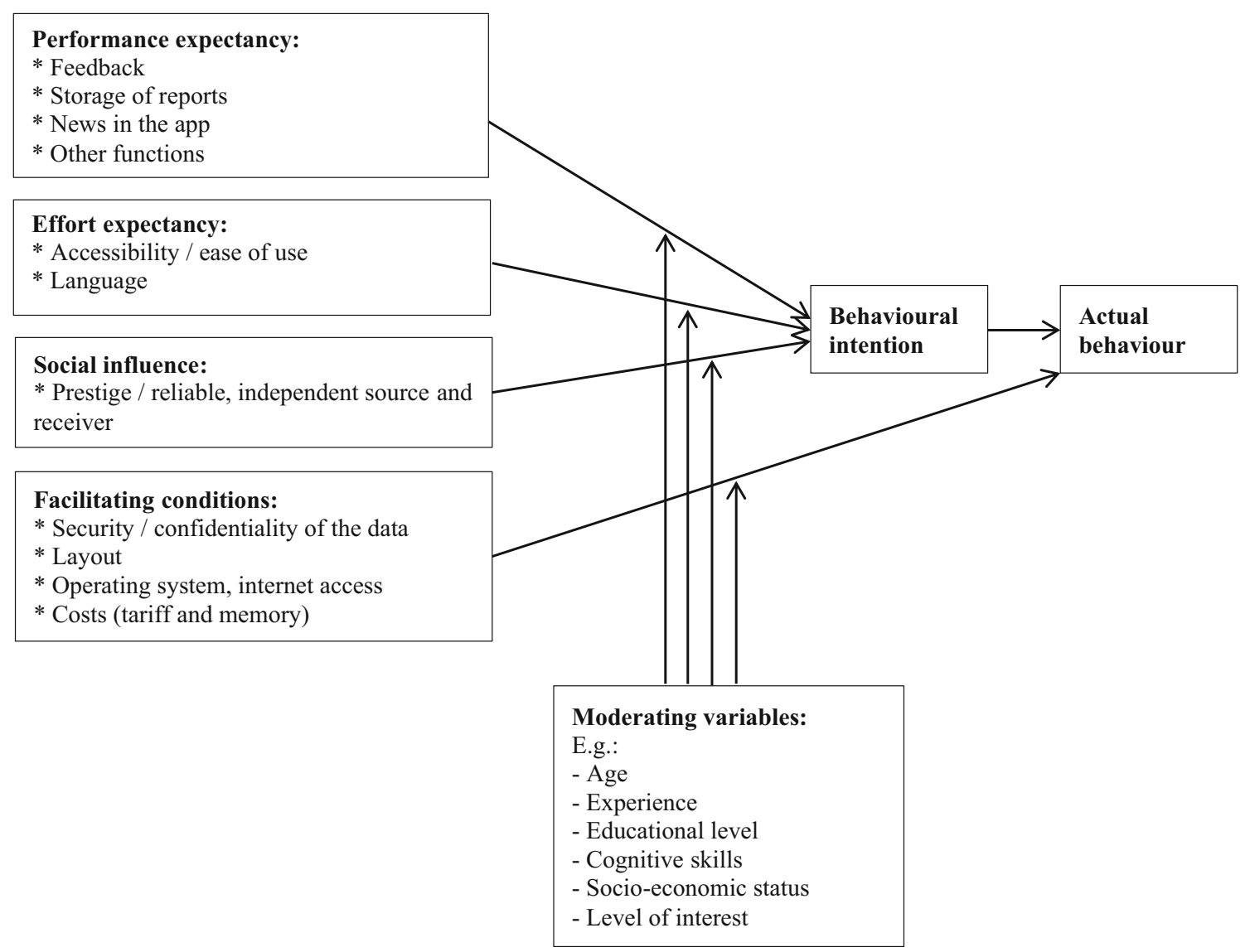

Fig. 2 Overview of the identified influencing factors and moderating variables of using an app for two-way risk communication

Some suggestions were offered for types of news items that would be of interest, such as newly identified ADRs of a drug, changes in the frequency of an ADR with a drug (e.g. from rarely to often), new drug-drug interactions, the issuance of Direct Healthcare Professional Communications (DHPCs or dear doctor letters), drugs taken off the market, newly marketed drugs or new applications of a drug, and changes in guidelines. However, not everyone was interested in all types of information. Some preferred to receive information for all drugs whereas others were interested only in those they took or prescribed themselves. Therefore, participants suggested app users should be able to personalise the type of information they wanted to receive news about.

News or information in the app may empower patients to communicate with their $\mathrm{HCP}$, for instance, by increasing patients' confidence in a symptom being an ADR.

"People who do not have the medical knowledge can have a look at their phone and they can see that it happens more often [a specific symptom], so I can discuss it with my doctor" (the Netherlands, Patient 3).

It might also help patients determine whether a symptom is an ADR, particularly if an HCP is not aware of the association.
Some patients indicated that they did not want to visit their doctor for every symptom they had experienced. The app may assist these patients to check whether these symptoms could be related to a drug they use.

3.1.1.4 Other Functions Several other functions were reported as useful for this type of app. An example was providing drug product information in the app. This may, however, negatively influence adherence since patients may not want to take a drug when they read about ADRs they may experience:

"I personally would use it before I take a medicine, also to see what sort of side effects I may get in case it's something severe I may not want to take the medication" (the UK, focus group with patients).

Other examples of functions that were seen as useful were the option to read about others' experiences with a drug and the option to add experiences (e.g. using a forum or providing a link to a forum), an overview of alternative drugs to the one where an ADR is experienced, alerts on quality defects (added value for pharmacists and prescribers), summary of guidelines, information about how to resolve/alleviate the ADR, interactions between drugs, and 
a prediction model presenting the likelihood of a symptom being an ADR after entering some information. The latter would be useful not only for HCPs but also for patients.

"If you were taking two medicines at the same time, how would you decide which side effect belonged to each drug?" (the UK, focus group with patients).

An option to store a list of (previously) prescribed drugs in the app could be useful in clinical practice and in ADR reports. In clinical practice, this could provide an up-todate drug list for HCPs that would be useful for both HCPs and patients (the Netherlands, HCP 4). In ADR reports, the drug list could be added to check whether co-medication might have impacted a reported ADR instead of a single causative drug entered by the patient (the Netherlands, Patient 4).

Discrepant opinions were given on, for instance, a reminder function in the app. Some participants thought it would be useful to have a reminder function to report an ADR, to go to an appointment with an HCP, or to take drugs (the UK, focus group with patients). Others found a reminder function for taking prescribed drugs irrelevant (the Netherlands, Patient 2).

\subsubsection{Effort Expectancy}

\subsubsection{Accessibility/Ease of Use An app was seen as an} easily accessible tool for two-way risk communication.

"You are immediately there where you have to be, you don't have to search on Google, you don't have to use paper and pen, you don't have to call" (the Netherlands, Patient 4).

However, participants indicated that information about the existence of the app would have to be widely circulated, as there are too many apps available.

"The problem with apps is that there are so many that you don't overlook it” (the Netherlands, HCP 5).

Some keywords describing a successful app were proposed: easy, simple, short, clear, logical. An app may increase the reporting of ADRs since time constraints were often mentioned by HCPs as an important factor for not reporting an ADR (the Netherlands, HCPs 3-8). Patients also mentioned time in relation to reporting an ADR.

"If I had to input all the information, if it took a lot of time I don't think I will [use it]" (the UK, focus group with patients).

In general, apps that do not meet the above-mentioned criteria were considered not likely to be used or to be used again.
Appreciated functions were those considered to facilitate ease of use, such as lists with, for instance, drug names that populate after entering a few letters, and options where answers can be selected (click on and/or drop-down options). For the latter, being able to input their answer or at least to be able to type in a different answer to the one offered was preferred. Inappropriate questions such as asking males if they were pregnant were seen as annoying and conveyed a feeling of not being taken seriously. A concern was raised that a very easy-to-use app might lead to over-reporting.

3.1.2.2 Language The language in the app was an important influencing factor for using it. The terminology in the app should be appropriate for HCPs and lay people.

"I think you should there, say, what we all understand in medical jargon is not understood by patients. [example given]. On the other hand, I do not want a thing [app] in which itching is mentioned [instead of pruritus] and that kind of" (the Netherlands, HCP 6).

Some HCPs as well as patients indicated that it would be useful to have the app in other languages.

"It is of course super luxury, we have a multicultural uh that it [the app] can also be used in English for instance. Like: translate this app in English" (the Netherlands, Patient 4).

\subsubsection{Social Influence}

\subsubsection{Prestige/Reliable, Independent Source and Recei-} ver A clear link of the app to an organisation with good standing or reputation would increase use of the app.

"People will trust it more if they know it is from [e.g.] NHS [National Health Service]" (the UK, focus group with patients).

In addition, it was important to the participants that ADR reports were sent to a reliable receiver, and that information would be provided by a reliable source. The source/receiver should be familiar to the potential app users. In addition, app use among patients may be increased if HCPs were to endorse it.

"[if the app was] endorsed by a doctor then it [the app] would be used a lot" (the UK, focus group with patients).

A topic that specifically came up during the focus group discussion with adolescents was whether parents should be able to view their child's ADR reports. In general, older children preferred parents not to have access and younger children wanted parents to be informed. 


\subsubsection{Facilitating Conditions}

\subsubsection{Security/Confidentiality of the Data A safe app was viewed as important.}

"I would like the app to have a security system to protect the personal data provided in each user profile" (Spain, focus group with patients and their carers).

However, most participants did not appear to be too concerned about security. They expected that no patientidentifiable details would be required and that the data would be handled confidentially.

"I should of course say that it should be a safe network etcetera, basically, you do not use the name of a patient, you use sex, date of birth, so I do not see any [security issues], no" (the Netherlands, HCP 1).

Storage of the report was seen as adequate when there would be no patient-identifiable information or when patient permission had been obtained (the Netherlands, HCP 8).

Participants with, or involved in, the care of patients with a rare disease, and adolescents seemed to be more concerned about the security of the app and the personal information in it. Some stated they would not use their real name but "put in a false name" (the UK, focus group with patients). Using login details each time they accessed the app was seen as necessary added protection of their data.

"People messing around on your phone can't log onto the account, so people can't just get into it and send random side effects" (the UK, focus group with patients).

However, some participants found it annoying to have to enter their login details each time they accessed the app and they were less concerned about the security of data stored in the app.

"There are no big medical secrets in it" (the Netherlands, Patient 3).

One suggestion made was that users would have the option of saving their login details on their personal devices.

3.1.4.2 Layout The layout of an app was seen as an important influencing factor for whether people would use the app and keep on using the app. In general, it was suggested never to use very bright colours. Such colours may, for instance, cause seizures in people with epilepsy. One indication was that if the colours, as well as font type and size, could be changed to one's own preferences this would facilitate the use of the app.
"I think, I assume [...], that you can change the colours yourself" (the Netherlands, Patient 2).

Participants indicated that the logo of the app should be recognisable and appealing. It should be clear from the logo what function the app has, clearly linking the app to the agency to which the ADR reports are sent.

3.1.4.3 Operating System, Internet Access It was suggested that the app should be available for all operating systems on mobile phones and tablets and that they should have been tested on each system before the app is launched.

"If you develop an app you have to do it for all three media. Apple, Android and Microsoft" (the Netherlands, HCP 4).

Adolescents were the only group who mentioned that it should be possible to enter an ADR report into the app while being 'off-line'. It should be possible to send the report later when 'on-line' and internet access became available. The motivation for that may be related to the costs, meaning that adolescents prefer to use freely available internet access on their mobile phones rather than activating mobile internet access for which they have to pay.

3.1.4.4 Costs (Tariff and Memory) Participants indicated that the app should be free of charge to stimulate use. A topic that was mentioned only by adolescents or HCPs treating adolescents was the concern about the amount of space the app took up on an individual's device.

"Will it clog up your phone", "take up loads of memory", "how cumbersome or big is the app", "what is the size of sending an average response (report). Is it in terms of just a few kilobytes?" (the UK, focus group with HCPs).

\subsection{User Characteristics}

Several user characteristics are described in the UTAUT model as moderating factors: age, gender, experience and voluntariness of use. Age and experience were also mentioned in our study. Participants indicated that they expected older people to be less likely to possess a mobile phone or tablet, to be more often visually impaired and to be generally less experienced with using apps than younger people. These factors could affect whether such patients will be reached through an app. It may also influence the preferences of potential users on the app lay-out (e.g. font size). In addition, it was mentioned that a user's previous experience with apps influences how easy they may find it to use this app. 
In our study, other characteristics were also mentioned as factors potentially influencing the use of the app. Characteristics such as educational level, cognitive skills, socio-economic status, ambition, duty, assertiveness, curiosity, level of interest and anxiety were mentioned as factors influencing not only being able to, daring to, or willing to report ADRs through an app, but also for possessing a mobile phone or tablet, and having the knowledge to use or look for information on an app.

\subsection{Behavioural Intention}

In general, participants were quite positive about an app for two-way risk communication. They liked the idea and indicated that there seems to be a need for it.

\subsection{Factors Influencing Reporting an ADR in General}

The focus group discussions and face-to-face interviews also revealed factors that may influence the reporting of an ADR in general. These influencing factors are related to knowledge, motivation and reporting of ADRs by patients.

\subsubsection{Knowledge}

There was a lack of knowledge among some patients about the possibility of being able to report an ADR at all.

"I didn't know that reporting an adverse reaction to medication was an option I had" (Spain, focus group with patients and their carers).

Some were familiar with ADR reporting but seemed unsure about which type of ADRs to report. This uncertainty was also observed in HCPs who said that they would report new ADRs, serious ADRs and any ADRs of a new drug. Participants were unsure if less severe ADRs required reporting.

"Is this severe enough to report?", "or they should say that you can report every ADR so that you don't have to think about whether this is something that they intend to collect" (the Netherlands, Patient 4).

They also stated that the goal of collecting ADRs should be clearly defined; for instance, whether it is about detecting rare ADRs or to quantify further the frequency of known ADRs.

\subsubsection{Motivation}

There was some discrepancy in motivation to report ADRs. Most patients and HCPs indicated that reporting an ADR should be based on intrinsic rather than extrinsic reasons.
"We are professionals so we have a certain faith so to speak" (the Netherlands, HCP 5).

"If I knew that the app that I was inputting all this information would benefit other people as well, then I would" (the UK, focus group with patients).

However, there were also some HCPs who suggested that extrinsic reasons might stimulate reporting. ADR reporting was stated to always be additional to the regular tasks and that it does not deliver any immediate benefits (the Netherlands, HCP 8).

One HCP mentioned that comparing the number of reported ADRs with colleagues (a kind of benchmarking) could stimulate reporting in the future. Also, HCPs' comparison with their previous reports may stimulate reporting.

"In the last months, I always did 20 reports, this time

it was 2, what is the cause of that? So that is also to stimulate to report" (the Netherlands, HCP 4).

\subsubsection{Reporting of ADRs by Patients}

It was mentioned that reporting an ADR might be less cumbersome for patients than for HCPs. Thus, patients could be asked more questions than HCPs (the Netherlands, HCP 7). However, this may be perceived differently by some patient groups as adolescents indicated that they did not like answering too many questions.

Some HCPs were worried about the quality of patient reports. Therefore, quality checks were suggested, such as inviting the HCP to also report the ADR for a particular patient or to ask additional questions in the patient report (e.g. whether it has been discussed with the HCP, whether other causes have been examined). Specifically, HCPs caring for patients with rare diseases indicated that reports should be validated by an HCP before they are sent elsewhere.

It was mentioned several times that linking the reports to a patient's clinical file would be useful. HCPs would immediately be up-to-date when opening a patient's clinical file. Moreover, HCPs indicated that they would be interested in what their patients had reported.

"If patients were reporting ADRs of drugs I'd started it would be useful to know. Definitely" (the UK, focus group with HCPs).

A concern HCPs had was that patients might report the ADR and take no further action.

"Don't want them to just report it and then carrying on with their life...don't want it to be a replacement for seeing their $\mathrm{HCP}$ " (the UK, focus group with HCPs). 
This concern seems justified since some adolescents mentioned that reporting an ADR, for instance through the app, might reduce the need for a verbal conversation with an $\mathrm{HCP}$, particularly in the case of embarrassing conditions (e.g. emotional problems) (the UK, focus group with patients).

\section{Discussion}

This study showed that HCPs and patients were generally positive about using an app for two-way risk communication. Actual use of the app would be influenced by the type of feedback given after an ADR has been reported; whether ADR reports can be stored safely; and what type of news is provided. Other, more general factors that may influence actual use are other functions of the app; ease of use, appropriate language and an attractive and possibly modifiable layout; the source (authority) behind the app; the security of the app; the operating systems on which the app is available; and the costs of the app in terms of tariff and memory. User characteristics such as age, experience, educational level, cognitive skills and personality characteristics were identified as potential moderating variables in the association between identified factors and the intention to use an app for two-way risk communication. Some identified factors (e.g. availability of other functions, security features) could both negatively and positively impact the use of an app and further studies are needed to determine how user characteristics influence this direction.

Some of the identified influencing factors apply particularly to an app for two-way risk communication. An important example of such a factor is the receiver to whom information is sent or the source of information provided through to the app. For effective risk communication, trust and/or confidence in the source/receiver are important [30]. Our results are similar to previous studies about app use with respect to influencing factors such as ease of use, language, layout, and costs [24, 31]. These can be seen as general influencing factors for using any app.

The finding that user characteristics may influence the use of an app for two-way risk communication is in accordance with the UTAUT model, in which several user characteristics are indicated as moderating variables. Elderly people, for instance, tend to adopt more slowly or reject technology [32] and may therefore have less intention to use an app. A previous study about health-app use also showed that user characteristics such as age, income and educational level influence the use of apps [33]. Further quantitative research is needed to assess whether all the characteristics mentioned in our study indeed influence the use of an app for two-way risk communication.
One of the aims of an app for two-way risk communication is to improve the communication of new safety information. Interestingly, some participants did not prefer an app for two-way risk communication in the sense that it contains both risk information and the option to report ADRs. They indicated that the app should just focus on one function, for instance ADR reporting. This finding might be due to the previously demonstrated negative association between the number of functions in an app and its usability [34]. Therefore, close attention should be paid to the usability of an app containing more than one function.

The other aim of an app for two-way risk communication is to increase the number of ADRs reported. A systematic review showed that a lack of knowledge on which ADRs to report (e.g. only serious or also less serious) is an important reason for the under-reporting of ADRs [35]. Although we did not systematically assess factors that influence the reporting of ADRs in general, it appears that, among others, a lack of knowledge on (1) the ability to report and (2) which type of ADRs to report influences reporting. Therefore, it might be important to provide clear guidance on which ADRs to report.

Previously, it has been shown that patients are not always certain whether a symptom could be an ADR [12-14, 36]. In addition, it has been indicated that patients may be uncertain about the exact drug causing the ADR [37]. The current study also shows this uncertainty since it was suggested by HCPs that a professional should always assess whether the entered drug or any of the co-medication caused the ADR. However, this may not be feasible in clinical practice.

The focus group discussions and face-to-face interviews revealed discrepancies in several aspects of an app for twoway risk communication; for instance, whether they want feedback and the type of feedback after reporting an ADR, the type of news in the app, and the use of login details. It is expected that such discrepancies apply to all apps and it would be prudent to develop an app that the user can personalise. Personalising options have been reported previously as an important factor to using an app [38].

\subsection{Strengths and Limitations}

A strength of our study was the inclusion of a variety of patients and HCPs from several European countries. As a result, it is expected that a wide range of influencing factors to use an app for two-way risk communication will have been covered. However, each patient or HCP group was assessed in a different country in which focus group discussions, face-to-face interviews or both methods were used. The included population may not fully represent the targeted patient/HCP population (e.g. there were more females) or indeed the whole of the potential target 
population for the Web-RADR app (e.g. all patients and HCPs). The generalisability of our findings is therefore limited, but as with most qualitative research, the findings are intended to generate hypotheses that need to be confirmed (e.g. in quantitative surveys). Moreover, for pragmatic reasons both face-to-face interviews and focus group discussions were used, whereas each method generally serves a different purpose. In our opinion, both methods were valuable in our study since potential factors influencing the use of an app for two-way risk communication were assessed and a prototype version of this app was tested. By using both methods, each task was thoroughly evaluated. Another limitation of the study relates to the use of the UTAUT model as a theoretical model since it has been tested to assess user acceptance of new technology in the workplace rather than technology in the healthcare sector. Although we were able to categorise each of the detected influencing factors to one of the themes of the UTAUT model, a previous study on health apps found a statistically significant association between behavioural intention and only one theme of the model (i.e. performance expectancy) [39]. Therefore, further research should be conducted to assess the relevance of the other domains and their interaction with user characteristics included in the model for the healthcare sector.

\subsection{Guidance for Future Studies}

Based on this study it appears that there may be differences (1) between patients and HCPs, (2) within patient and HCP groups, and/or (3) between countries regarding factors potentially influencing the use of an app for two-way risk communication. Therefore, additional, preferably quantitative studies such as survey studies in large groups of patients and HCPs are advocated to increase knowledge about the influencing factors on using such an app. In future studies, several countries should include the same patient population and/or HCP group to be able to assess whether differences are due to variations among patients and/or HCP groups or among countries. We already stated that additional research could assess the influence of user characteristics on using an app for two-way risk communication. Several other suggestions for further research are as follows.

In our study, patients with a rare disease or HCPs treating patients with a rare disease and adolescents seemed to be more concerned about the security of the app than those with type 2 diabetes and their HCPs. This may be due to the fact that an individual with a rare disease can be more easily identified in healthcare data than someone without a rare disease [40]. A previous study among adolescents also found that high importance was attached to a safe app [31]. Further studies may determine whether diverse patient populations and HCP groups differ in their opinions on the importance of app security or whether such differences are country-related.

The cost of the app in terms of space on a device was mentioned only as a factor that negatively influences app use by adolescents or HCPs treating adolescents in our study. It might be that this is a particular concern for younger persons or for people in the UK.

Time constraints were mentioned as an important factor for not reporting ADRs. This finding has also been shown in a systematic review [35]. An app for two-way risk communication may reduce the time needed to complete an ADR report, but future studies are needed to test whether an app will indeed increase the reporting of ADRs by patients and HCPs.

An app for two-way risk communication may also have unintended effects such as increased non-adherence and patients not visiting their HCP. Awareness of the possibility of unintended effects is important and future studies should be conducted to assess such effects.

A quantitative study will also be useful to assess how many people are interested in an app for two-way risk communication rather than having separate apps for each function; the number of people interested in some other specific functions in an app (e.g. a reminder function); the type of news people want to receive in such an app; whether or not people want to receive feedback after sending a report, and the type of feedback; and preferences for presenting a patient-reported ADR to someone's HCP and/or parent in the case of adolescents.

\section{Conclusions}

This study identified factors that could influence the use of a mobile app for two-way risk communication. These factors concern feedback; storage of ADR reports; and the type of news provided in the app. More general factors concern other functions of the app; ease of use; appropriate language and attractive layout; the source of the information in the app and the receiver of information provided through the app; the security and costs of the app; and the operating systems on which the app is available. Further studies are needed to quantify the influencing factors and determine the differences among patients and HCPs, between patient and HCP groups, and between countries with respect to influencing factors to use a mobile app for two-way risk communication. Also, further studies are needed to determine how user characteristics influence the direction (positive or negative) of the identified factors on actual use of the app. Assessing influencing factors is relevant to foster uptake and continued use of an app. 
Acknowledgements The authors wish to thank Kyra McKenna and Lucas Baptista of Epidemico, Ltd. (Ireland), mobile developers for the apps studied in this article.

Web-RADR work package $3 \mathrm{~b}$ representatives: Raphael van Eemeren, Karin Hace, Sandra Fernandes, Faiza Afzal, Dennis Costello.

Author Contributions All authors contributed to the development and formulation of the research question. STdV and PGMM acquired the data in the Netherlands, conducted the analysis of the Dutch data and interpreted the Dutch data. LW and AS acquired the data in the UK, conducted the analysis of the UK data and interpreted the UK data. CLR acquired the data in Spain, conducted the analysis of the Spanish data and interpreted the Spanish data. All authors contributed to the interpretation of the overall data. STdV wrote the manuscript. LW, AS, FH, CLR and PGMM reviewed and edited the manuscript. All authors have read and approved the final manuscript.

\section{Compliance with Ethical Standards}

Conflict of interest Sieta T. de Vries, Lisa Wong, Alastair Sutcliffe, François Houÿez, Carmen Lasheras Ruiz, and Peter G.M. Mol declare that they have no conflicts of interest.

Ethical approval All procedures performed in this study were carried out in accordance with the ethical standards of the institutional and/or national research committee and with the 1964 Helsinki declaration and its later amendments or comparable ethical standards. Ethical approval for this study was sought as determined by each country's local ethics committee policy. In Spain, ethical approval was obtained from the Fundació Sant Joan de Déu. In the UK, ethical approval was obtained from the University College London. In the Netherlands, a waiver for full ethical approval was obtained from the University Medical Center Groningen (reference number M15.172178). Informed consent was obtained from all participants.

Funding The Web-RADR project has received support from the Innovative Medicine Initiative Joint Undertaking (http://www.imi. europa.eu) under Grant Agreement no. 115632-resources of which are composed of financial contributions from the European Union's Seventh Framework Programme FP7/2007-2013 and EFPIA companies in kind contribution, http://www.imi.europa.eu, UK.

Open Access This article is distributed under the terms of the Creative Commons Attribution-NonCommercial 4.0 International License (http://creativecommons.org/licenses/by-nc/4.0/), which permits any noncommercial use, distribution, and reproduction in any medium, provided you give appropriate credit to the original author(s) and the source, provide a link to the Creative Commons license, and indicate if changes were made.

\section{References}

1. Downing NS, Aminawung JA, Shah ND, Krumholz HM, Ross JS. Clinical trial evidence supporting FDA approval of novel therapeutic agents, 2005-2012. JAMA. 2014;311(4):368-77. doi:10. 1001/jama.2013.282034.

2. Raine J, Wise L, Blackburn S, Eichler HG, Breckenridge A. European perspective on risk management and drug safety. Clin Pharmacol Ther. 2011;89(5):650-4. doi:10.1038/clpt.2011.28.

3. Stricker BH, Psaty BM. Detection, verification, and quantification of adverse drug reactions. BMJ. 2004;329(7456):44-7.
4. Segal ES, Valette C, Oster L, Bouley L, Edfjall C, Herrmann P, et al. Risk management strategies in the postmarketing period: safety experience with the US and European bosentan surveillance programmes. Drug Saf. 2005;28(11):971-80.

5. Blenkinsopp A, Wilkie P, Wang M, Routledge PA. Patient reporting of suspected adverse drug reactions: a review of published literature and international experience. Br J Clin Pharmacol. 2007;63(2):148-56.

6. Anker SD, Agewall S, Borggrefe M, Calvert M, Jaime Caro J, Cowie MR, et al. The importance of patient-reported outcomes: a call for their comprehensive integration in cardiovascular clinical trials. Eur Heart J. 2014;35(30):2001-9. doi:10.1093/eurheartj/ ehu205.

7. Basch E, Reeve BB, Mitchell SA, Clauser SB, Minasian LM, Dueck AC, et al. Development of the National Cancer Institute's patient-reported outcomes version of the common terminology criteria for adverse events (PRO-CTCAE). J Natl Cancer Inst. 2014;106(9):dju244. doi:10.1093/jnci/dju244.

8. Matos C, Harmark L, van Hunsel F. Patient reporting of adverse drug reactions: an international survey of national competent authorities' views and needs. Drug Saf. 2016;39(11):1105-16. doi:10.1007/s40264-016-0453-6.

9. Belton KJ, Lewis SC, Payne S, Rawlins MD, Wood SM. Attitudinal survey of adverse drug reaction reporting by medical practitioners in the United Kingdom. Br J Clin Pharmacol. 1995;39(3):223-6.

10. Lorimer S, Cox A, Langford NJ. A patient's perspective: the impact of adverse drug reactions on patients and their views on reporting. J Clin Pharm Ther. 2012;37(2):148-52. doi:10.1111/j. 1365-2710.2011.01258.x.

11. Vallano A, Cereza G, Pedros C, Agusti A, Danes I, Aguilera C, et al. Obstacles and solutions for spontaneous reporting of adverse drug reactions in the hospital. Br J Clin Pharmacol. 2005;60(6):653-8. doi:10.1111/j.1365-2125.2005.02504.x.

12. Chaipichit N, Krska J, Pratipanawatr T, Uchaipichat V, Jarernsiripornkul N. A qualitative study to explore how patients identify and assess symptoms as adverse drug reactions. Eur J Clin Pharmacol. 2014;70(5):607-15. doi:10.1007/s00228-014-1653-6.

13. de Vries ST, Mol PG, de Zeeuw D, Haaijer-Ruskamp FM, Denig P. Development and initial validation of a patient-reported adverse drug event questionnaire. Drug Saf. 2013;36(9):765-77. doi:10.1007/s40264-013-0036-8.

14. de Vries ST, Haaijer-Ruskamp FM, de Zeeuw D, Denig P. The validity of a patient-reported adverse drug event questionnaire using different recall periods. Qual Life Res. 2014;23(9): 2439-45. doi:10.1007/s11136-014-0715-7.

15. Yamamoto M, Kubota K, Okazaki M, Dobashi A, Hashiguchi M, Doi $\mathrm{H}$, et al. Patients views and experiences in online reporting adverse drug reactions: findings of a national pilot study in Japan. Patient Prefer Adher. 2015;9:173-84. doi:10.2147/PPA.S75761.

16. European Medicines Agency. Guideline on good pharmacovigilance practices (GVP) Module XV—safety communication [Internet]. Available from: http://www.ema.europa.eu/docs/en_GB/ document_library/Scientific_guideline/2013/01/WC500137666. pdf. Accessed 5 Aug 2016.

17. Goldman SA. Communication of medical product risk: how effective is effective enough? Drug Saf. 2004;27(8):519-34.

18. Piening S, Reber KC, Wieringa JE, Straus SM, de Graeff PA, Haaijer-Ruskamp FM, et al. Impact of safety-related regulatory action on drug use in ambulatory care in the Netherlands. Clin Pharmacol Ther. 2012;91(5):838-45. doi:10.1038/clpt.2011.308.

19. Morris L. Communicating drug risks to patients [Internet]. Available from: http://www.medscape.org/viewarticle/581013_2. Accessed 5 Aug 2016.

20. Ahmed H, Naik G, Willoughby H, Edwards AGK. Communicating risk. BMJ. 2012;344:e3996. doi:10.1136/bmj.e3996. 
21. Ghosh R, Lewis D. Aims and approaches of Web-RADR: a consortium ensuring reliable ADR reporting via mobile devices and new insights from social media. Expert Opin Drug Saf. 2015;14(12):1845-53. doi:10.1517/14740338.2015.1096342.

22. Damodaran L. User involvement in the systems design processa practical guide for users. Behav Inf Technol [Internet]. 1996;15(6):363-77. Available from: http://www.tandfonline. com/doi/pdf/10.1080/014492996120049. Accessed 5 Aug 2016.

23. Jeon E, Park HA. Factors affecting acceptance of smartphone application for management of obesity. Healthc Inform Res. 2015;21(2):74-82. doi:10.4258/hir.2015.21.2.74.

24. Scheibe M, Reichelt J, Bellmann M, Kirch W. Acceptance factors of mobile apps for diabetes by patients aged 50 or older: a qualitative study. Med 2 0. 2015;4(1):e1. doi:10.2196/med20. 3912.

25. Cordova D, Bauermeister JA, Fessler K, Delva J, Nelson A, Nurenberg R, et al. A community-engaged approach to developing an mHealth HIV/STI and drug abuse preventive intervention for primary care: a qualitative study. JMIR Mhealth Uhealth. 2015;3(4):e106. doi:10.2196/mhealth.4620.

26. Grindrod KA, Li M, Gates A. Evaluating user perceptions of mobile medication management applications with older adults: a usability study. JMIR Mhealth Uhealth. 2014;2(1):e11. doi:10. 2196/mhealth.3048.

27. Morse JM. Theoretical saturation. In: The SAGE encyclopedia of social science research methods [Internet]. Available from: http:// sk.sagepub.com/reference/download/socialscience/n1011.pdf. Accessed 5 Aug 2016.

28. Braun V, Clarke V. Using thematic analysis in psychology. Qual Res Psychol. 2006;3(2):77-101.

29. Venkatesh V, Morris MG, Davis GB, Davis FD. User acceptance of information technology: toward a unified view. MIS Q. 2003;27(3):425-78.

30. Slovic P, Peters E, Grana J, Berger S, Dieck GS. Risk perception of prescription drugs: results of a national survey. Drug Inf J. 2007;41(1):81-100.
31. Kenny R, Dooley B, Fitzgerald A. Developing mental health mobile apps: exploring adolescents' perspectives. Health Inf J. 2016;22(2):265-75. doi:10.1177/1460458214555041.

32. Steele R, Lo A, Secombe C, Wong YK. Elderly persons' perception and acceptance of using wireless sensor networks to assist healthcare. Int J Med Inform. 2009;78(12):788-801. doi:10.1016/ j.ijmedinf.2009.08.001.

33. Krebs P, Duncan DT. Health app use among US mobile phone owners: a national survey. JMIR Mhealth Uhealth. 2015;3(4): e101. doi:10.2196/mhealth.4924.

34. Arnhold M, Quade M, Kirch W. Mobile applications for diabetics: a systematic review and expert-based usability evaluation considering the special requirements of diabetes patients age 50 years or older. J Med Internet Res. 2014;16(4):e104. doi:10.2196/ jmir.2968.

35. Lopez-Gonzalez E, Herdeiro MT, Figueiras A. Determinants of under-reporting of adverse drug reactions: a systematic review. Drug Saf. 2009;32(1):19-31. doi:10.2165/00002018-20093201000002.

36. Britten N. Medication errors: the role of the patient. Br J Clin Pharmacol. 2009;67(6):646-50. doi:10.1111/j.1365-2125.2009.03421.x.

37. de Vries ST, Haaijer-Ruskamp FM, de Zeeuw D, Denig P. Construct and concurrent validity of a patient-reported adverse drug event questionnaire: a cross-sectional study. Health Qual Life Outcomes. 2014;12:103. doi:10.1186/s12955-014-0103-6.

38. Tang J, Abraham C, Stamp E, Greaves C. How can weight-loss app designers' best engage and support users? A qualitative investigation. Br J Health Psychol. 2015;20(1):151-71. doi:10. 1111/bjhp.12114.

39. Yuan S, Ma W, Kanthawala S, Peng W. Keep using my health apps: discover users' perception of health and fitness apps with the UTAUT2 model. Telemed J E Health. 2015;21(9):735-41. doi:10.1089/tmj.2014.0148.

40. Mascalzoni D, Paradiso A, Hansson M. Rare disease research: breaking the privacy barrier. Appl Transl Genom. 2014;3(2): 23-9. doi:10.1016/j.atg.2014.04.003. 